\title{
The effects of linear and logarithmic data transformations upon observed patterns of lingual vibrotactile sensitivity
}

\author{
KAL M. TELAGE \\ School of Allied Health, Ithaca College, Ithaca, New York 14850 \\ and \\ DONALD J. FUCCI \\ School of Hearing and Speech Sciences, Ohio University, Athens, Ohio 45701
}

\begin{abstract}
Vibrotactile thresholds were obtained from the anterior midline surface of the tongue on three groups of normal-speaking young adults. A different frequency condition was employed for each of the groups tested. Threshold values were initially recorded in millivolts and subsequently converted to microns of displacement and decibels. Data comparisons showed a differential response to frequency for both kinds of transformations. This finding was discussed in terms of its implications concerning the nature and type of mechanoreceptors present in the cutaneous surface tissue of the tongue. Some suggestions for further research in this area were made.
\end{abstract}

Recent investigations using sinusoidal stimuli to measure lingual vibrotactile sensitivity have produced quantifiable threshold data at different test frequencies. In general, these studies have employed similar experimental procedures in which the measurement unit initially determined threshold values in millivolts. Data were then converted to microns of vibratory displacement using a linear transformation or to decibels by way of a logarithmic transformation. The two conversion procedures have tended to emphasize different aspects of the sensory data which have been reflected in two distinct patterns of threshold sensitivity across frequency. A result has been that researchers have drawn different conclusions concerning the nature and function of pressure receptors beneath the tongue's surface which mediate vibrotactile sensation.

Specifically, thresholds reported in microns of displacement have shown variations in sensitivity strongly suggesting that mechanoreceptors in the lingual surface consistently summate energy in the frequency range of 80 to $400 \mathrm{~Hz}$ (Telage, Fucci, \& Arnst, 1972; Kelly, Note 1; Telage, Note 2). Threshold data expressed in decibels (reference 1 micron RMS) have produced flat threshold curves through the same frequency range, implying that peripheral receptors in the tongue's surface are not differentially sensitive to changes in mechanical vibration (Fucci, 1972; Fucci, Hall, \& Weiner, 1971; Verrillo, 1966a).

Although it is anticipated that linear and logarithmic data transformations might emphasize different aspects of the threshold results, it does not seem plausible that a differential frequency response would be obtained for

James L. Bruning sponsors this paper and takes full editorial responsibility for its content. micron values and obscured for decibel values. Instead, it seems reasonable to expect neural elements in the cutaneous tissue of the tongue to demonstrate consistent levels of threshold excitation to mechanical stimulation which would be reflected in both micron and decibel measurement values.

\section{METHOD}

The equipment used in this study has been described in a previous publication (Fucci, 1972). The stimulus unit included a sine-wave generator, frequency counter, electronic switch (designed to maintain a sine-wave configuration), amplifier, variable attenuator, and electromagnetic vibrator. The pulsed vibratory signal generated had a 50\% duty cycle (on $1 \mathrm{sec}$ and off $1 \mathrm{sec})$ with a rise and decay time of $100 \mathrm{msec}$. The measurement unit consisted of an accelerometer, cathode follower, microphone amplifier, and voltmeter. A white-noise generator was used to present auditory masking at 100-110 dB SPL to subjects through TDH-39 headphones.

Subjects in the present study ranged in age from 18 to 36 years and reported no history of speech, sensory, and/or motor impairments. Lingual vibrotactile thresholds were obtained for 150 subjects at 200,300 , and $400 \mathrm{~Hz}$. The frequencies selected fall within a range tested by each of the investigations previously reviewed. Fifty subjects were tested at each respective frequency using an ascending psychophysical method of limits. Subjects were required to respond by depressing an indicator switch when they detected the stimulus. The median of three separate trials was accepted as each subject's threshold. Thresholds were initially measured in millivolts. They were then changed to displacement in microns using a standard $\mathrm{g}$ formula for acceleration conversion and decibel values (re. 1 micron RMS), for comparative purposes.

\section{RESULTS AND DISCUSSION}

Mean threshold values and standard deviations are presented in Table 1. A comparison between the 
Table 1

Means and Standard Deviations for Vibrotactile Threshold Data Obtained from the Anterior Midline Surface of the Tongue

\begin{tabular}{lccc}
\hline & \multicolumn{3}{c}{ I'requency } \\
\cline { 2 - 4 } & 200 & 300 & 400 \\
\hline $\begin{array}{l}\text { Micron Data } \\
\text { Mean }\end{array}$ & .445 & .177 & .243 \\
SD & .320 & .103 & .124 \\
Decibel Data & & & \\
Mean & -8.9 & -16.0 & -13.2 \\
SD & 5.8 & 4.1 & 4.3 \\
\hline
\end{tabular}

Note $-N=150$ (50 at each frequency).

threshold data reported in microns of vibratory displacement (linear transformation) and the same data expressed in decibels (logarithmic transformation) shows a similar threshold pattern across the frequencies tested. Differential threshold response characterized by maximum sensitivity at $300 \mathrm{~Hz}$ and minimum sensitivity at $200 \mathrm{~Hz}$ are observed for both micron and decibel values.

Differences between mean vibrotactile threshold values reported in microns for each pair of frequency combinations were analyzed by dependent $t$ tests. A statistically significant difference at the .05 level of confidence was found for each comparison. These data are presented in Table 2. The nature of the ratio measure (decibels) did not allow for similar statistical treatment.

One possible explanation for the flat responses previously reported by studies expressing threshold values in decibels and the sloping curves reported by investigators who transformed data into microns may be related to the number of subjects tested. The decibel study samples were 3 subjects, 5 subjects, and 10 subjects, respectively. (Fucci, 1972; Fucci, Hall, \& Weiner, 1971; Verrillo, 1966b). Investigations reporting thresholds in microns tested 110 subjects, 30 subjects, and 50 subjects, respectively (Telage, Fucci, \& Arnst, 1972; Kelly, Note 1; Telage, Note 2). Fucci, Hall, and Weiner (1971) have speculated that logarithmic data transformations compress the dynamic range which tends somewhat to flatten threshold curves. It may be that the use of very small samples in the decibel studies cited did not produce initial millivolt values which approximated normal variability. In this situation, transforming these data to decibels might compress the results to a degree showing a flat response across frequency. The fact that this did not occur in the present study might be attributed to a larger sample which provided a broader range of thresholds. This explanation implies the need to use adequately large experimental samples for physiological studies from which normative implications may be drawn.

These findings appear to support the hypothesis that peripheral mechanoreceptors in the lingual surface demonstrate consistent levels of threshold excitation which are observable in both micron and decibel data transformations. The data further suggest that the vibratory sense is served by a distinct set of primary afferents which are differentially sensitive to the high frequency range $(200-400 \mathrm{~Hz})$ used in this study. This frequency function is similar to response characteristics of receptors in the glabrous skin of the palmar surface which are reported by Verrillo (1966b), who concluded that the Pacinian corpuscle was the underlying cutaneous transducer mediating vibratory sensation.

A neurophysiological basis for these results has been reported by Mountcastle, Talbot, Sakata, and Hyvarinen (1968). In their study of neural mechanism responses of monkeys to high-frequency $(80-400 \mathrm{~Hz})$ vibrotactile stimulation, they found evidence that Pacinian corpuscle cortical neurons receive relayed afferents only from Pacinian peripheral fibers activated by mechanical stimulation of the hand. In addition, these investigators reported a wide convergence of peripheral Pacinian corpuscle fibers upon Pacinian corpuscle cortical neurons. This last finding suggests a possible neurophysiological basis for summation effects observed by Verrillo $(1965,1966 a)$ and further supports the argument that Pacinian corpuscles may be the exteroceptive receptors which are specifically sensitive to mechanical vibration.

It appears that the results of this investigation and the ensuing discussion pose questions relative to lingual vibrotactile sensitivity. First, are neural receptors in the anterior surface of the tongue transducers for mechanically induced vibratory stimuli? Second, is this neural system similar to that which is found in glabrous cutaneous tissue? Affirmative support for these questions is provided by the research cited in this paper in that it demonstrates significantly different threshold levels across frequency irrespective of the final data transformation.

The frequency-intensity parameters have been studied to a greater extent for lingual tissue than other variables which might provide more definitive information. Other research might assess possible spatial and temporal summation effects upon threshold patterns derived from the tongue. This might be accomplished by obtaining lingual vibrotactile thresholds using a number of

Table 2

A Comparison of Mean Differences in Microns for Vibrotactile Thresholds Obtained at 200, 300, and $400 \mathrm{~Hz}$

\begin{tabular}{ccc}
\hline Frequencies $(\mathrm{Hz})$ & Threshold Values & $\mathrm{t}$ Vilues \\
\hline 200 & .445 & $5.63^{*}$ \\
300 & .177 & \\
200 & .445 & $4.17^{*}$ \\
400 & .243 & \\
300 & .177 & $-2.87^{*}$ \\
400 & .243 & \\
\hline
\end{tabular}

Note $-N=150$

*Significant at the 05 level 
different lingual contactors which vary in area. It would also be possible to develop an experimental procedure which establishes oral sensory thresholds for vibrotactile stimuli using different pulse durations. Such data could provide more extensive information concerning the response characteristics of neural transducers which mediate the vibratory sense for the tongue. Furthermore, it is anticipated that this kind of data might help to identify the nature of these receptors and the manner by which the nervous system discriminates among vibrotactile stimuli.

\section{REFERENCE NOTES}

1. Kelly, D. H. Oral vibrotactile sensation: An evaluation of children exhibiting normal articulation and children exhibiting defective articulation. Unpublished doctoral dissertation, Ohio University, 1973.

2. Telage, K. M. Measurement considerations in determining lingual vibrotactile thresholds: An analysis of interval and ratio scales. Paper presented at the New York State Speech and Hearing Association Convention, 1974.

\section{REFERENCES}

Fucci, D. J. Oral vibrotactile sensation: An evaluation of normal and defective speakers. Joumal of Speech and Hearing Research, 1972, 15, 179-184.

Fucci, D. J., Hall, D. E., \& Weiner, F. F. Normative study of oral and nonoral structures using vibrotactile stimuli. Perceptual and Motor Skills, 1971, 33, 1099-1105.

Mountcastle, V. B., Talbot, W. H., Sakata, H., \& Hyvarinen, J. Cortical neuronal mechanisms in flutter-vibration studied in unanesthetized monkeys. Neuronal periodicity and frequency discrimination. Joumal of Neurophysiology, 1968, 31, 452-484.

Telage, K. M., Fucci, D. J., \& Arnst, D. J. Normative study of oral vibrotactile sensitivity. Perceptual and Motor Skills, 1972, $35,671-676$

Verrillo, R. T. Temporal summation in vibrotactile sensitivity. Joumal of the Acoustical Society of America, 1965, 37, 843-846.

Verrillo, R. T. Effect of spatial parameters on the vibrotactile threshold. Journal of Experimental Psychology, 1966, 71 . 570-575. (a)

Verrillo, R. T. Specificity of a cutaneous receptor. Perception \& Psychophysics, 1966, 1, 149-153. (b)

(Received for publication April 21, 1975.) 